\title{
Image Database Design Based on 9D-SPA Representation for Spatial Relations
}

\author{
Po-Whei Huang and Chu-Hui Lee
}

\begin{abstract}
Spatial relationships between objects are important features for designing a content-based image retrieval system. In this paper, we propose a new scheme, called 9D-SPA representation, for encoding the spatial relations in an image. With this representation, important functions of intelligent image database systems such as visualization, browsing, spatial reasoning, iconic indexing, and similarity retrieval can be easily achieved. The capability of discriminating images based on 9D-SPA representation is much more powerful than any spatial representation method based on Minimum Bounding Rectangles or centroids of objects. The similarity measures using 9D-SPA representation provide a wide range of fuzzy matching capability in similarity retrieval to meet different user's requirements. Experimental results showed that our system is very effective in terms of recall and precision. In addition, the 9D-SPA representation can be incorporated into a two-level index structure to help reduce the search space of each query processing. The experimental results also demonstrated that, on average, only 0.1254 percent $\sim 1.6829$ percent of symbolic pictures (depending on various degrees of similarity) were accessed per query in an image database containing 50,000 symbolic pictures.
\end{abstract}

Index Terms_-Image database, spatial relations, similarity retrieval, 9D-SPA, visualization.

\section{INTRODUCTION}

pictorial database plays an important role in many A applications including geographical information systems, computer aided design, office automation, medical image archiving, and trademark picture registration. The traditional approach to image database design is to use textual descriptions to annotate images and then store annotations in a text-based data base management system. Searching for desired images is equivalent to searching for the associated annotations. This approach is too tedious and labor-intensive. Moreover, the key words used in annotations or query descriptions are too subjective and may become inadequate, especially when the number of images in the database increase tremendously.

Content-based image retrieval (CBIR) is the current trend of designing image database systems as opposed to textbased image retrieval [7], [11], [14], [18], [23], [24], [25], [27]. The features used in content-based image retrieval can be roughly divided into two categories: the low-level visual features (such as color, texture, and shape) and the highlevel features (such as pairwise spatial relationships between objects). Some examples of content-based image retrieval systems are QBIC [8], Virage [1], Retrieval Ware [29], VisualSEEK [26], WaveGuide [17], and Photobook [21]. They allow users to retrieve similar pictures from a large image database based on low-level visual features. On the other hand, there is also a large group of researchers

- P.W. Huang is with the Department of Computer Science, National Chung-Hsing University, Taichung 40227, Taiwan, Republic of China.

E-mail: powhei.huang@nhust.edu.tw.

- C.H. Lee is with the Department of Information Management, Chaoyang University of Technology, 168 Gifeng E. Rd., Wufeng, Taichung County, Taiwan, Republic of China. E-mail: chlee@cyut.edu.tw.

Manuscript received 20 June 2002; revised 18 Feb. 2004; accepted 18 Feb. 2004.

For information on obtaining reprints of this article, please send e-mail to: tkde@computer.org, and reference IEEECS Log Number 116826. emphasizing image retrieval based on spatial relationships between objects [3], [4], [5], [10], [15], [16], [20], [22], [28]. In this paper, we only concentrate on iconic picture retrieval based on spatial relations where the number of icons in the database are fixed and each object in a picture must match an icon.

The method of representing images is one of the major concerns in designing an image database system. The representation method for an image should capture the knowledge about the image's content as much as possible. One way of representing an image is to construct a symbolic picture for that image which in turn is encoded into a 2D-string [5]. The 2D string representation method opened up a new approach to spatial reasoning, picture indexing, and similarity retrieval. There are many followup research works based on the concept of $2 \mathrm{D}$ string such as 2D C-string [15], [16], and 2D $\mathrm{C}^{+}$-string [9].

An ideal representation method for symbolic pictures should provide image database systems with many important functions such as similarity retrieval, visualization, browsing, spatial reasoning, and picture indexing. In this paper, we propose a new scheme for encoding spatial relations called 9-Direction SPanning Area (9D-SPA) representation method. Using the 9D-SPA representation, we can easily accomplish the following system design goals:

1. Iconic pictures can be easily reconstructed from 9D-SPA representations for visualization.

2. Flexibility and accuracy in similarity retrieval can be achieved at the same time through a set of coarse-tofine similarity measures.

3. The 9D-SPA representation can be incorporated into an efficient index structure so that the search space will be restricted to a relatively small portion of the database for improving retrieval efficiency. 
The remainder of this paper is organized as follows: In Section 2, previous research works about knowledge representations for spatial relations are discussed. In Section 3, the 9D-SPA representation is introduced. In Section 4, we propose a visualization method for 9D-SPA representation. The algorithm of decoding the 9D-SPA representation for picture reconstruction is also presented in this section. Spatial reasoning and interpretation based on a 9D-SPA representation are presented in Section 5. In Section 6, we define a set of similarity measures for fuzzy matching. We also introduce an index structure based on 9D-SPA representation to facilitate image retrieval. The similarity retrieval algorithm is presented in this same section. The experimental results for demonstrating the efficiency and effectiveness of our approach are presented in Section 7. Finally, conclusions are given in the last section.

\section{Overview of Spatial Knowledge REPRESENTATION}

Binary spatial relationships between objects have been identified as one of the most important features for describing the contents of images [6]. For example, a query such as "finding all the pictures containing a house to the east of a tree" relies on spatial relations to retrieve the desired pictures. Different kinds of spatial knowledge representations have been proposed so far. Chang et al. [5] proposed the $2 \mathrm{D}$ string as a spatial knowledge representation to capture the spatial information about the content of a picture. The fundamental ideal of 2D string is to project the objects of a picture along the $x$ and $y$-directions to form two strings representing the relative positions of objects in the $x$ and $y$-axis, respectively. Since a 2D string preserves the spatial relationships between any two objects in a picture, it has the advantage of facilitating spatial reasoning. Moreover, since a query picture [6] can also be represented as a 2D string, the problem of similarity retrieval becomes a problem of $2 \mathrm{D}$ string subsequence matching.

Jungert [12], Chang et al. [4], and Jungert and Chang [13] extended the idea of 2D strings to form 2D G-strings by introducing several new spatial operators to represent more relative positional relationships among objects of a picture. The 2D G-string representation embeds more information about spatial relationships between objects and, thus, facilitates spatial reasoning about sizes and relative positions of objects.

Following the same concept, Lee and Hsu [15] proposed the 2D C-string representation based on a special cutting mechanism. Since the number of subparts generated by this new cutting mechanism is reduced significantly, the lengths of the strings representing pictures are much shorter while still preserving the spatial relationships among objects. The 2D C-string representation is more economical in terms of storage space efficiency and navigation complexity in spatial reasoning. The 2D $\mathrm{C}^{+}$-string representation [9] extended the $2 \mathrm{D}$ C-string representation by adding relative metric information about the picture to the strings. As a consequence, reasoning about relative sizes and locations of

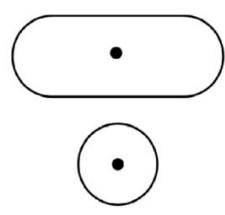

(a)

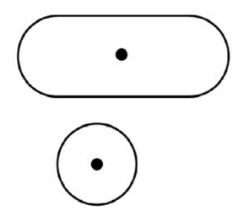

(b)

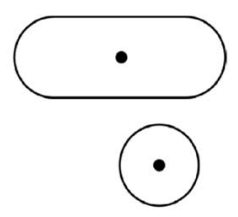

(c)
Fig. 1. Spatial reasoning is too sensitive in the 9DLT representation.

objects, as well as the relative distance between objects in a symbolic picture becomes possible.

Chang [3] proposed a structure called 9DLT to encode the spatial relationships between objects in terms of nine directions. Since the 9DLT method uses centroid to represent the position of an object, such a representation is too sensitive in spatial reasoning. For example, the spatial relationships between the two objects shown in Figs. 1a, 1b, and 1c are all different in 9DLT representation; however, they seem not too much different in human visual perception.

The representation of spatial relations proposed by Zhou and Ang [28] combines the nine directional relations proposed in 9DLT with the five topological relations, namely, disjoint, meet, partly_overlap, contain, and inside. The topological relation can record the $2 \mathrm{D}$ relationship between any two nonzero-sized objects with irregular shapes and, therefore, makes spatial reasoning more accurate as compared to using MBR or centroid to represent an object. However, Zhou and Ang's method still has the problem with being too sensitive when reasoning about directional relations.

Instead of combining the nine directional relations with the five topological relations, the 2D-PIR proposed by Nabil et al. [19], [20] combines the 13 projection interval relations with the topological relations. Although 2D-PIR seems particularly useful in similarity retrieval, it did not provide any picture reconstruction mechanism for visualization. Besides, incorporating 2D-PIR into any indexing structure is difficult. Thus, similarity retrieval based on 2D-PIR becomes inefficient if the volume of images in the database increases.

\section{9D-SPA REPRESENTATION}

To represent a picture using our method, the picture has to be preprocessed first. We assume that the objects in a picture can be identified by some image segmentation and object recognition procedures. Various techniques of image segmentation and object recognition can be found in [2].

Suppose that a picture $P$ contains $n$ objects $\left(O_{1}, O_{2}, \ldots, O_{n}\right)$. Then, the 9D-SPA representation of $P$ can be encoded as a set of 4-tuples: $R=\left\{\left(O_{i j}, D_{i j}, D_{j i}, T_{i j}\right) \mid \forall O_{i}, O_{j} \in P\right.$, and $1 \leq i$ $<j \leq n\}$, where $O_{i j}$ is the code for object-pair $\left(O_{i}, O_{j}\right), D_{i j}$ is the code for the direction-relation between objects $O_{i}, O_{j}$ with $O_{j}$ as the reference object, $D_{j i}$ is the code for the directionrelation between $O_{i}$ and $O_{j}$ with $O_{i}$ as the reference object, and $T_{i j}$ is the code for the topological relation between $O_{i}$ and $O_{j}$. It is obvious that the number of 4-tuples in $R$ is $\frac{n(n-1)}{2}$. 
TABLE 1

The Codes for Nine Neighborhood Areas of MBR of $O_{j}$

\begin{tabular}{|l|l|l|}
\hline $\begin{array}{l}\text { Area 4: } \\
(00001000)_{2}=8\end{array}$ & $\begin{array}{l}\text { Area 3: } \\
(00000100)_{2}=4\end{array}$ & $\begin{array}{l}\text { Area 2: } \\
(00000010)_{2}=2\end{array}$ \\
\hline Area 5: & $\begin{array}{l}\left.\text { Area 0:(MBR of } O_{j}\right) \\
(00010000)_{2}=16\end{array}$ & $\begin{array}{l}\text { Area 1: } \\
(00000000)_{2}=0\end{array}$ \\
\hline $\begin{array}{l}\text { Area 6: } \\
(00100000)_{2}=32\end{array}$ & $\begin{array}{l}\text { Area 7: } \\
(01000000)_{2}=64\end{array}$ & $\begin{array}{l}\text { Area 8: } \\
(10000000)_{2}=128\end{array}$ \\
\hline
\end{tabular}

Let $O_{i}$ be the $i$ th object in the image database $(1 \leq i \leq n)$. We assign integer $i$ to object $O_{i}$ as its object number. Then, $O_{i j}$ is called the object-pair code for object-pair $\left(O_{i}, O_{j}\right)$. Given two objects $O_{i}$ and $O_{j}$, we can easily compute the object-pair code $O_{i j}$ using the following formula:

$$
O_{i j}=\frac{(j-1)(j-2)}{2}+i .
$$

To obtain the two object numbers $i$ and $j$ from $O_{i j}$ (or to decode $O_{i j}$ ), we use the formula $i=O_{i j}-\frac{a(a+1)}{2}$, where $a$ is the largest integer such that $\frac{a(a+1)}{2}<O_{i j}$ and $j=a+2$.

$D_{i j}$ represents the value assigned to the directional relationship between objects $O_{i}$ and $O_{j}$ with $O_{j}$ as the reference object. The value of $D_{i j}$ is determined by the following procedure. First, we find the Minimal Bounding Rectangle (MBR) for reference object $O_{j}$. Then, we extend the four boundaries of this MBR horizontally and vertically until they cut the whole picture into nine neighborhood areas and then assign each area a binary code as shown in Table 1 . The value of $D_{i j}$ is determined by the formula $D_{i j}=\sum_{k=1}^{8} b_{k} w_{k}$, where $w_{k}$ is the binary code of neighborhood area $k ; b_{k}=1$ if object $O_{i}$ overlaps area $k$, otherwise, $b_{k}=0$. The value of $T_{i j}$ indicates the topological relationship between objects $O_{i}$ and $O_{j}$. Possible values assigned to topological relations are: 0 (stands for "disjoint"), 1 (stands for "meet"), 2 (stands for "partly_overlap"), 3 (stands for "cover"), and 4 (stands for "contain" or "inside").

Let us look at the two pictures shown in Figs. $2 a$ and $2 b$. Assume that object $B$ is the reference object in both pictures. Then, in Fig. 2a, the code for $D_{A B}$ is

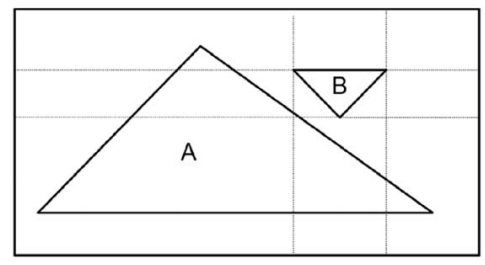

(a)
$(00001000+00010000+00100000+01000000+10000000)_{2}$ $=248$

and the code for $T_{A B}$ is 0 . In Fig. $2 \mathrm{~b}$, the code for $D_{A B}$ is

$$
\begin{aligned}
& (00000001+00000010+00100000+01000000+10000000)_{2} \\
& =227
\end{aligned}
$$

and the code for $T_{A B}$ is 0 . In $2 \mathrm{D}^{*}$-string representations, the pictures in Figs. $2 \mathrm{a}$ and $2 \mathrm{~b}$ are not distinguishable because they have the same spatial representation (i.e., $\mathrm{A} \% \mathrm{~B}$ in both $x$ and $y$-directions). However, we can easily tell the difference between them by using 9D-SPA representation because $D_{A B}$ in Fig. 2a is 248, while $D_{A B}$ in Fig. $2 \mathrm{~b}$ is 227. Moreover, from $D_{A B}=248=(11111000)_{2}$, we can easily determine that object $A$ spans five neighborhood areas of object $B$, namely, the northwest, the west, the southwest, the south, and the southeast neighborhood areas as shown in Fig. 2a. Similarly, from $D_{A B}=227=(11100011)_{2}$, we can easily determine that object $A$ spans another different five neighborhood areas of object $B$, namely, the northeast, the east, the southeast, the south, and the southwest neighborhood areas as shown in Fig. $2 b$.

\section{Visualization}

In this section, we will describe how to construct a symbolic picture from a 9D-SPA representation. The capability of reconstructing pictures can support visualization and database browsing. Our goal is to construct a symbolic picture from a 9D-SPA representation so that each object in the picture is approximated by its MBR and the spatial relationships among all objects are also preserved. In other words, we need to determine the order of begin and endpositions of each object along the $x$ and $y$-axis. Because the relative positions of objects in the $x$-axis are independent of their relative positions in the $y$-axis, the reconstruction procedure can be performed separately for each direction.

The projection positions of objects in a picture along the $x$ and $y$-directions can be represented by two directed acyclic graphs $G_{x}=\left(V_{x}, E_{x}\right)$ and $G_{y}=\left(V_{y}, E_{y}\right)$, called the Spatial Relation Graphs. A node in $V_{x}$ contains one or more variables whose values indicating either the begin or endpositions of objects in the $x$-direction. Similarly, a node in $V_{y}$ contains one or more variables whose values indicating either the begin or end-positions of objects in the $y$-direction. All variables in the same node will be assigned the same position value. For example, if $\overrightarrow{u v} \in E_{x}, b_{i}$ and $e_{j}$ are two variables in node $u$ while $b_{k}$ is a variable in node $v$, then $b_{i}=e_{j}<b_{k}$.

Given a 9D-SPA representation, we can construct two spatial relation graphs $G_{x}$ and $G_{y}$, which in turn will be used

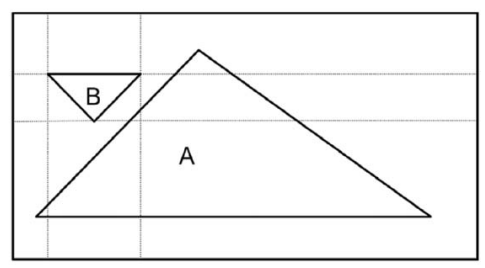

(b)

Fig. 2. Pictures (a) and (b) are not distinguishable in all $2 \mathrm{D}^{*}$-string representations. However, the difference can be easily determined by the $9 \mathrm{D}-\mathrm{SPA}$ representation. 
to construct a corresponding symbolic picture. The algorithm shown below allows us to create spatial relation graphs from a 9D-SPA representation. For convenience of explanation, we use the notations below to describe the algorithm.

- $G_{x}$ (or $\left.G_{y}\right)$ : the spatial relation graph for the $x$-direction (or $y$-direction).

- $b_{i}^{x}$ (or $e_{i}^{x}$ ): the begin-position (or end-position) of object $O_{i}$ in the $x$-direction.

- $b_{j}^{y}$ (or $e_{j}^{y}$ ): the begin-position (or end-position) of object $O_{j}$ in the $y$-direction.

Algorithm: Generating spatial relation graphs.

Input: A 9D-SPA representation $R$.

Output: $G_{x}$ and $G_{y}$.

1. $V_{x} \leftarrow \emptyset ; E_{x} \leftarrow \emptyset ; V_{y} \leftarrow \emptyset ; E_{y} \leftarrow \emptyset$.

2. $\forall\left(O_{i j}, D_{i j}, D_{j i}, T_{i j}\right) \in R$,

a. Decode $O_{i j}$ to obtain two object numbers $i$ and $j$ as described in Section 3.

b. Create four nodes $B_{i}^{x}, E_{i}^{x}, B_{j}^{x}, E_{j}^{x}$ containing variables $b_{i}^{x}, e_{i}^{x}, b_{j}^{x}, e_{j}^{x}$, respectively. Create another four nodes $B_{i}^{y}, E_{i}^{y}, B_{j}^{y}, E_{j}^{y}$ containing variables $b_{i}^{y}, e_{i}^{y}, b_{j}^{y}, e_{j}^{y}$, respectively.

c. Compare $D_{i j}$ with the binary codes of the 8 neighborhood areas of $O_{j}$ to find the projection relations among the begin-positions and endpositions of $O_{i}$ and $O_{j}$ along the $x$-direction. Then, create the edges corresponding to these projection relations among the nodes $B_{i}^{x}, B_{j}^{x}, E_{i}^{x}$, $E_{j}^{x}$ and add them to $G_{x}$.

d. Similarly, find the projection relations among the begin-positions and end-positions of $O_{i}$ and $O_{j}$ along the $y$-direction. Then, create the edges corresponding to these projection relations among the nodes $B_{i}^{y}, B_{j}^{y}, E_{i}^{y}, E_{j}^{y}$ and add them to $G_{y}$.

e. Exchange indices $i$ and $j$, then repeat Steps c-d to process $D_{j i}$.

3. For each cycle $C$ in $G_{x}$, use node $d$ to replace all the nodes in $C$ such that

a. $d$ has all the variables originally contained in each node in $C$.

b. If there is an in-edge from a node not in $C$ to any node in $C$, then this edge is redirected to $d$.

c. If there is an out-edge from a node in $C$ to any node not in $C$, then this edge is modified to originate from $d$.

4. Remove all cycles from $G_{y}$ by a similar procedure as described in Step 3.

5. Return $G_{x}$ and $G_{y}$.

Once two spatial relation graphs $G_{x}$ and $G_{y}$ have been created from a 9D-SPA representation, we can easily construct a symbolic picture from $G_{x}$ and $G_{y}$ as follows:

Algorithm: Picture reconstruction.

Input: $G_{x}$ and $G_{y}$.

Output: A symbolic picture.

1. Find the set of nodes $S_{1} \subseteq V_{x}$ from $G_{x}$ such that $\forall u \in S_{1}, u$ has no in-edges.

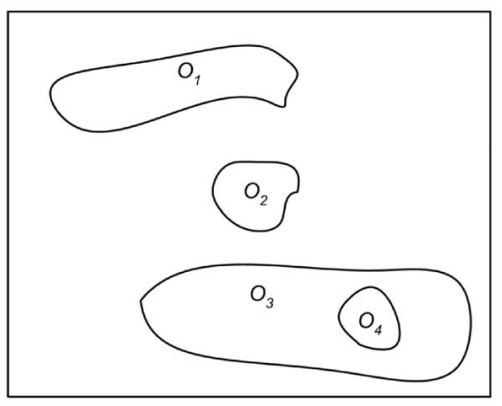

Fig. 3. A picture containing four objects.

2. $\quad i \leftarrow 1$.

3. $\forall u \in S_{1}$, assign value $i$ to all the position variables contained in node $u$ and mark $u$ as "inspected."

4. Find the immediate successors of the nodes in $S_{1}$ such that they have not been inspected. Let this set be $S_{2}$. If $S_{2}=\emptyset$, then go to Step 6 .

5. $i \leftarrow i+1 ; S_{1} \leftarrow S_{2}$. Go to Step 3 .

6. Assign values to all position variables contained in $G_{y}$ by a similar procedure as described in Steps 1-5.

7. For each object $O_{i}$, draw the rectangle with $\left(b_{i}^{x}, b_{i}^{y}\right)$ and $\left(e_{i}^{x}, e_{i}^{y}\right)$ as the coordinates of the bottom-left and the top-right corners, respectively.

We use the picture shown in Fig. 3 to illustrate our reconstruction algorithm. The 9D-SPA representation for this image is shown in the first column of Table 2. From each tuple of this 9D-SPA representation, we can determine the nodes and the edges to be added into spatial relation graphs $G_{x}$ and $G_{y}$. For example, if a tuple $(1,12,64,0)$ in the first row of Table 2 is given, the two involved objects are $O_{1}$ and $O_{2}$. Thus, the nodes to be added into $G_{x}$ are $B_{1}^{x}, E_{1}^{x}, B_{2}^{x}$, and $E_{2}^{x}$. The nodes to be added into $G_{y}$ are $B_{1}^{y}, E_{1}^{y}, B_{2}^{y}$, and $E_{2}^{y}$. For $D_{12}=12$, the edges to be added into $G_{x}$ are $\overrightarrow{B_{1}^{x} B_{2}^{x}}$, $\overrightarrow{B_{2}^{x} E_{1}^{x}}, \overrightarrow{E_{1}^{x} E_{2}^{x}}$. The edges to be added into $G_{y}$ are $\overrightarrow{B_{2}^{y} E_{2}^{y}}, \overrightarrow{E_{2}^{y} B_{1}^{y}}$, $\overrightarrow{B_{1}^{y} E_{1}^{y}}$. Similarly, for $D_{21}=64$, the edges to be added into $G_{x}$ are $\overrightarrow{B_{1}^{x} B_{2}^{x}}, \overrightarrow{B_{2}^{x} E_{2}^{x}}, \overrightarrow{E_{2}^{x} E_{1}^{x}}$, and the edges to be added into $G_{y}$ are $\overrightarrow{B_{2}^{y} E_{2}^{y}}, \overrightarrow{E_{2}^{y} B_{1}^{y}}, \overrightarrow{B_{1}^{y} E_{1}^{y}}$. As a consequence, the spatial relation graphs $G_{x}$ and $G_{y}$ constructed are shown in Figs. $4 \mathrm{a}$ and $4 \mathrm{c}$, respectively. Notice that there exists a cycle in $G_{x}$. After removing this cycle from $G_{x}$, a new graph is formed as shown in Fig. 4b. From Figs. 4b and 4c, we assign a number to each node of the graphs according to their precedence order. By assigning the node number to all variables contained in that node, we have $b_{1}^{x}=1, b_{3}^{x}=2, b_{2}^{x}=3$, $e_{1}^{x}=e_{2}^{x}=4, b_{4}^{x}=5, e_{4}^{x}=6, e_{3}^{x}=7$, and $b_{3}^{y}=1, b_{4}^{y}=2, e_{4}^{y}=3$, $e_{3}^{y}=4, b_{2}^{y}=5, e_{2}^{y}=6, b_{1}^{y}=7, e_{1}^{y}=8$. Based on the above information, we can easily reconstruct a picture shown in Fig. 5. Notice that the original picture (Fig. 3) and the symbolic picture reconstructed from the 9D-SPA representation (Fig. 5) are the same in terms of the spatial 
TABLE 2

Constructing the Spatial Relation Graphs $G_{x}$ and $G_{y}$

\begin{tabular}{|c|c|c|c|}
\hline 4-tuple & object-pair & $D_{i j}$ & Edges added to $G_{x}$ and $G_{y}$. \\
\hline \multirow[t]{2}{*}{$(1,12,64,0)$} & $(1,2)$ & $D_{12}=00001100$ & $\overrightarrow{B_{1}^{x} B_{2}^{x}}, \overrightarrow{B_{2}^{x} E_{1}^{x}}, \overrightarrow{E_{1}^{x} E_{2}^{x}}, \overrightarrow{B_{2}^{y} E_{2}^{y}}, \overrightarrow{E_{2}^{y} B_{1}^{y}}, \overrightarrow{B_{1}^{y} E_{1}^{y}}$ \\
\hline & & $D_{21}=01000000$ & $\overrightarrow{B_{1}^{x} B_{2}^{x}}, \overrightarrow{B_{2}^{x} E_{2}^{x}}, \overrightarrow{E_{2}^{x} E_{1}^{x}}, \overrightarrow{B_{2}^{y} E_{2}^{y}}, \overrightarrow{E_{2}^{y} B_{1}^{y}}, \overrightarrow{B_{1}^{y} E_{1}^{y}}$ \\
\hline \multirow[t]{2}{*}{$(2,12,192,0)$} & $(1,3)$ & $D_{13}=00001100$ & $\overrightarrow{B_{1}^{x} B_{3}^{x}}, \overrightarrow{B_{3}^{x} E_{1}^{x}}, \overrightarrow{E_{1}^{x} E_{3}^{x}}, \overrightarrow{B_{3}^{y} E_{3}^{y}}, \overrightarrow{E_{3}^{y} B_{1}^{y}}, \overrightarrow{B_{1}^{y} E_{1}^{y}}$ \\
\hline & & $D_{31}=11000000$ & $\overrightarrow{B_{1}^{x} B_{3}^{\vec{x}}}, \overrightarrow{B_{3}^{x} E_{1}^{\mathrm{x}}}, \overrightarrow{E_{1}^{x} E_{3}^{\mathrm{x}}}, \overrightarrow{B_{3}^{y} E_{3}^{y}}, \overrightarrow{E_{3}^{y} B_{1}^{y}}, \overrightarrow{B_{1}^{y} E_{1}^{y}}$ \\
\hline \multirow[t]{2}{*}{$(3,4,224,0)$} & $(2,3)$ & $D_{23}=00000100$ & $\overrightarrow{B_{3}^{x} B_{2}^{x}}, \overrightarrow{B_{2}^{x} E_{2}^{\vec{x}}}, \overrightarrow{E_{2}^{x} E_{3}^{\vec{x}}}, \overrightarrow{B_{3}^{y} E_{3}^{y}}, \overrightarrow{E_{3}^{y} B_{2}^{y}}, \overrightarrow{B_{2}^{y} E_{2}^{y}}$ \\
\hline & & $D_{32}=11100000$ & $\overrightarrow{B_{3}^{x} B_{2}^{x}}, \overrightarrow{B_{2}^{x} E_{2}^{x}}, \overrightarrow{E_{2}^{x} E_{3}^{x}}, \overrightarrow{B_{3}^{y} E_{3}^{y}}, \overrightarrow{E_{3}^{y} B_{2}^{y}}, \overrightarrow{B_{2}^{y} E_{2}^{y}}$ \\
\hline \multirow[t]{2}{*}{$(4,8,128,0)$} & $(1,4)$ & $D_{14}=00001000$ & $\overrightarrow{B_{1}^{x} E_{1}^{\vec{x}}}, \overrightarrow{E_{1}^{x} B_{4}^{\mathrm{x}}}, \overrightarrow{B_{4}^{x} E_{4}^{\vec{z}}} \overrightarrow{B_{4}^{y} E_{4}^{\vec{y}}}, \overrightarrow{E_{4}^{y} B_{1}^{y}}, \overrightarrow{B_{1}^{y} E_{1}^{y}}$ \\
\hline & & $D_{41}=10000000$ & $\overrightarrow{B_{1}^{x} E_{1}^{\vec{x}}}, \overrightarrow{E_{1}^{x} B_{4}^{x}}, \overrightarrow{B_{4}^{x} E_{4}^{x}}, \overrightarrow{B_{4}^{y} E_{4}^{y}}, \overrightarrow{E_{4}^{y} B_{1}^{y}}, \overrightarrow{B_{1}^{y} E_{1}^{y}}$ \\
\hline \multirow[t]{2}{*}{$(5,8,128,0)$} & $(2,4)$ & $D_{24}=00001000$ & $\overrightarrow{B_{2}^{x} E_{2}^{x}}, \overrightarrow{E_{2}^{x} B_{4}^{x}}, \overrightarrow{B_{4}^{x} E_{4}^{x}}, \overrightarrow{B_{4}^{y} E_{4}^{y}}, \overrightarrow{E_{4}^{y} B_{2}^{y}}, \overrightarrow{B_{2}^{y} E_{2}^{\vec{y}}}$ \\
\hline & & $D_{42}=10000000$ & $\overrightarrow{B_{2}^{x} E_{2}^{\vec{x}}}, \overrightarrow{E_{2}^{x} B_{4}^{\mathrm{x}}}, \overrightarrow{B_{4}^{x} E_{4}^{\mathrm{t}}}, \overrightarrow{B_{4}^{y} E_{4}^{y}}, \overrightarrow{E_{4}^{y} B_{2}^{y}}, \overrightarrow{B_{2}^{y}, E_{2}^{y}}$ \\
\hline \multirow[t]{2}{*}{$(6,255,0,4)$} & $(3,4)$ & $D_{34}=11111111$ & $\overrightarrow{B_{3}^{x} B_{4}^{x}}, \overrightarrow{B_{4}^{x} E_{4}^{x}}, \overrightarrow{E_{4}^{x} E_{3}^{x}}, \overrightarrow{B_{3}^{y} B_{4}^{y}}, \overrightarrow{B_{4}^{y} E_{4}^{y}}, \overrightarrow{E_{4}^{y} E_{3}^{y}}$ \\
\hline & & $D_{43}=00000000$ & $\overrightarrow{B_{3}^{x} B_{4}^{\vec{x}}}, \overrightarrow{B_{4}^{x} E_{4}^{\vec{x}}}, \overrightarrow{E_{4}^{x} E_{3}^{x}}, \overrightarrow{B_{3}^{y} B_{4}^{y}}, \overrightarrow{B_{4}^{y} E_{4}^{y}}, \overrightarrow{E_{4}^{y} E_{3}^{y}}$ \\
\hline
\end{tabular}

relationships (both directional and topological) between any two objects.

\section{Spatial Reasoning and Interpretation}

Since a 9D-SPA representation already contains all pairwise spatial relations between objects of a picture, the spatial reasoning and interpretation process based on 9D-SPA representation becomes very simple and efficient. Given a 4-tuple $\left(O_{i j}, D_{i j}, D_{j i}, T_{i j}\right)$ of a 9D-SPA representation, the topological relationship such as "disjoint," "meet," "partly_overlap" between objects $O_{i}$ and $O_{j}$ is clearly indicated by the value of $T_{i j}$. To decide whether a topological relation is either "contain" or "inside," we use the following rules: If $D_{i j}=$ "11111111" and $T_{i j}=4$, then $O_{i}$ contains $O_{j}$; if $D_{j i}=$ "11111111" and $T_{i j}=4$, then $O_{i}$ is inside of $O_{j}$. Furthermore, if $D_{i j}=$ $D_{j i}=$ "00000000," then $O_{i}$ coincides with $O_{j}$.

The 9D-SPA representation can also decide another three topological relationships which are rarely discussed, but occur quite often in the real world as shown in Fig. 6:

1. Object $O_{j}$ is surrounded by object $O_{i}$ if $T_{i j}=0$ and $D_{i j}=$ "11111111."

2. Object $O_{j}$ is tightly enclosed by object $O_{i}$ if $T_{i j}=1$ and $D_{i j}=$ "11111111."

3. Object $O_{j}$ is overlapped with object $O_{i}$ at the inside boundary of $O_{i}$ if $T_{i j}=2$ and $D_{i j}=$ "11111111."

To determine the directional spatial relationship between object $O_{i}$ and $O_{j}$ from $\left(O_{i j}, D_{i j}, D_{j i}\right)$ is quite obvious. Notice

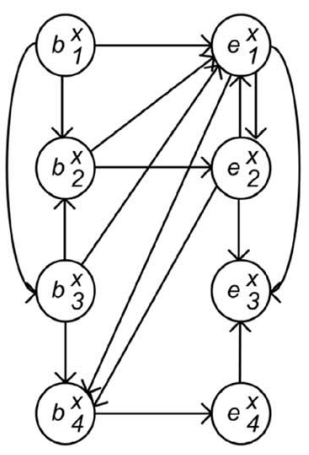

(a)

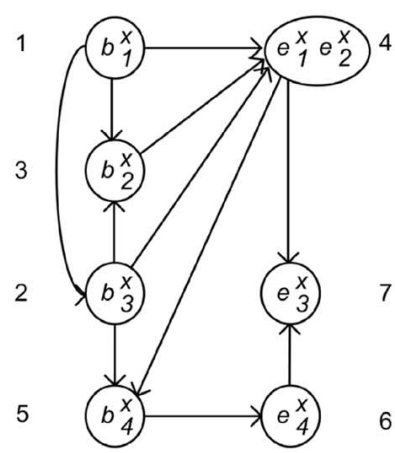

(b)

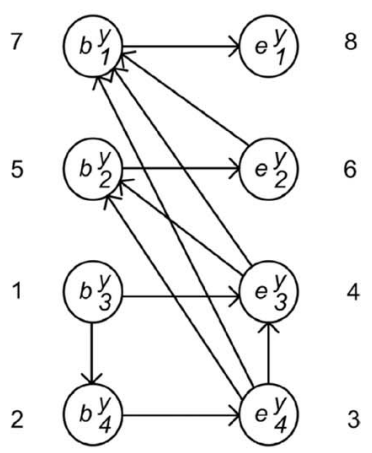

(c)

Fig. 4. Spatial relation graphs $G_{x}$ and $G_{y}$ for the picture shown in Fig. 3. (a) $G x$ with a cycle, (b) $G x$, and (c) $G_{y}$. 


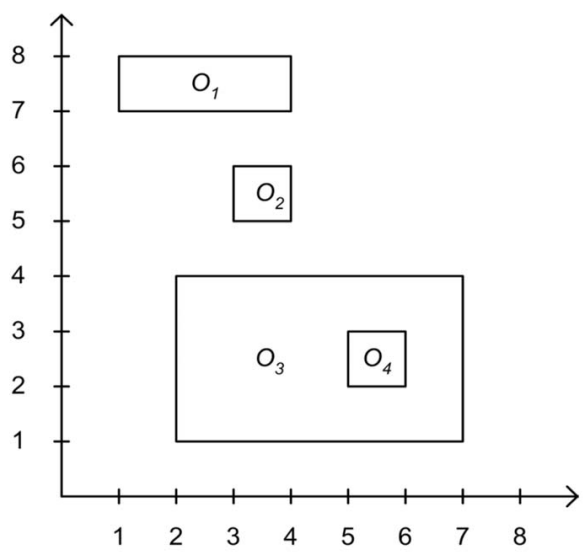

Fig. 5. The symbolic picture reconstructed from $G_{x}$ and $G_{y}$ shown in Fig. 4.

that $D_{i j}$ indicates the spatial location of object $O_{i}$ viewed from object $O_{j}$, and $D_{j i}$ indicates the spatial location of $O_{j}$ viewed from object $O_{i}$. Without loss of generality, we use $O_{j}$ as the reference object to interpret $D_{i j}$.

1. $O_{i}$ is to the east of $O_{j}$ if $D_{i j}=$ "00000001."

2. $O_{i}$ is to the northeast of $O_{j}$ if $D_{i j}=$ "00000010."

3. $O_{i}$ is to the north of $O_{j}$ if $D_{i j}=$ "00000100."

4. $O_{i}$ is to the northwest of $O_{j}$ if $D_{i j}=$ "00001000."

5. $O_{i}$ is to the west of $O_{j}$ if $D_{i j}=$ "00010000."

6. $O_{i}$ is to the southwest of $O_{j}$ if $D_{i j}=$ "00100000."

7. $O_{i}$ is to the south of $O_{j}$ if $D_{i j}=$ "01000000."

8. $O_{i}$ is to the southeast of $O_{j}$ if $D_{i j}=$ "10000000."

For more complicated cases, it is not so easy to precisely describe the directional relationship between $O_{i}$ and $O_{j}$ if $D_{i j}$ has more than one nonzero bit. Since $D_{i j}$ implies the spanning neighborhood areas of object $O_{i}$ viewed from object $O_{j}$, we simply enumerate all the neighborhood areas spanned by $O_{i}$ to indicate the directional relationship between $O_{i}$ and $O_{j}$. For example, if $D_{i j}=$ "00111100," then we say that $O_{i}$ spans the north, the northwest, the west, and the southwest neighborhood areas of object $O_{j}$. If $D_{i j}=$ "00000011," then object $O_{i}$ spans the east and the northeast neighborhood areas of object $O_{j}$. As we can see, this kind of description and interpretation is general as well as precise for directional spatial relationship between nonzero-sized objects.

In general, the spatial relationship between any two objects is not symmetric. The spatial relationship between objects $O_{i}$ and $O_{j}$ viewed from $O_{j}$ (i.e., $D_{i j}$ ) may be different from the spatial relationship between the same two objects viewed from $O_{i}$ (i.e., $D_{j i}$ ). For example, object $O_{i}$ spans the east neighborhood area of object $O_{j}$; however, object $O_{j}$ may span the northwest, the west, and the southwest neighborhood areas of object $O_{i}$. In other words, both $D_{i j}$ and $D_{j i}$ are needed in order to determine the spatial relationship between objects $O_{i}$ and $O_{j}$ correctly.

\section{Similarity RETRIEVAL}

In similarity retrieval, the user must present a query picture to match with the database images. One convenient way of presenting a query is to draw a sketch diagram called query picture [6] in which all objects must match some icons in the database. The task of image retrieval is to measure the similarity between the query picture and the database picture, then retrieve relevant pictures from the database. Usually, the user may not remember the exact spatial relationships among the objects in a desired picture. To accommodate this flexibility, the system should provide the user with a set of coarse-to-fine similarity measures to measure the difference between the query picture and the database picture. With a coarse measure, we allow the retrieved images to be slightly different from the query picture. However, the retrieved images still meet the user's requirements in term of user's visual perception.

\subsection{Similarity Measures}

Before giving detailed definitions for similarity measures, we introduce the following notations first:

- $\quad R_{p}\left(\right.$ or $\left.R_{q}\right)$ : the 9D-SPA representation for picture $p$ (or $q$ ).

- $\quad t^{p}\left(\right.$ or $\left.t^{q}\right)$ : a tuple in $R_{p}$ (or $R_{q}$ ).

- $\quad t . O$ : the object-pair code of tuple $t$.

- $t . D_{1}$ : the directional relation-code, with $O_{j}$ as the reference object, of tuple $t$.

- $\quad$ t. $D_{2}$ : the directional relation-code, with $O_{i}$ as the reference object, of tuple $t$.

- $\quad t . T$ : the topological relation-code of tuple $t$.

- $t . D_{1}(j)$ (or $t . D_{2}(j)$ ): the $j$ th bit of the binary code of t. $D_{1}$ (or $\left.t . D_{2}\right)$.

- $\quad x(k)$ : the $k$ th bit of the binary code $x$ (or $y$ ).

Let $R_{q}=\left\{t_{1}^{q}, t_{2}^{q}, \ldots, t_{m}^{q}\right\}$ and $R_{p}=\left\{t_{1}^{p}, t_{2}^{p}, \ldots, t_{n}^{p}\right\}$ be the 9DSPA representations for query picture $q$ and database picture $p$, respectively. Let $\varepsilon$ be a relation from $R_{q}$ to $R_{p}$. Without loss of generality, we assume that $\varepsilon\left(t_{i}^{q}\right) . O=t_{i}^{p}$. $O$ for $1 \leq i \leq k$ with $k \leq \min \{m, n\}$.

The basic idea of defining a directional similarity measure is explained as follows: Assume that $A$ and $B$ are

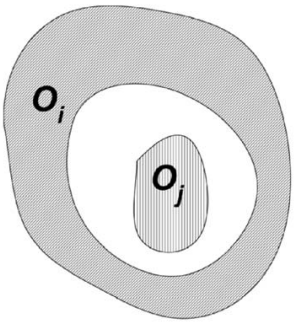

(a)

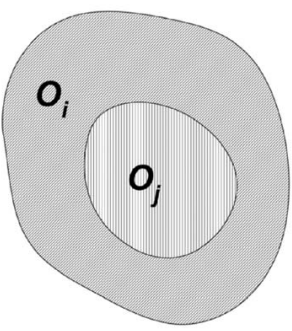

(b)

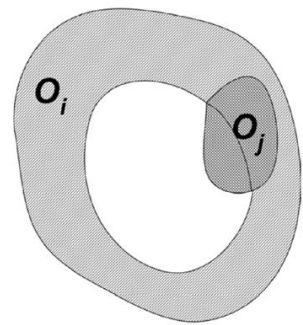

(c)

Fig. 6. Three special topological relationships. 


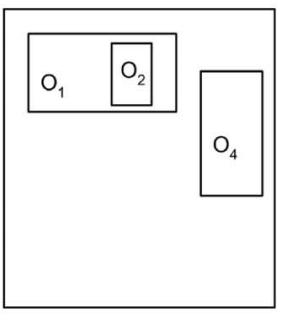

$p_{1}$

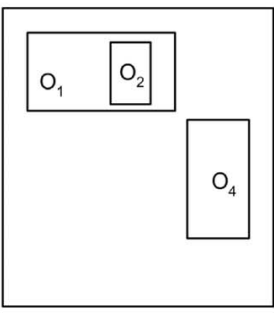

$p_{2}$

Fig. 7. A similarity measurement example.

two target objects represented by the same icon and $C$ is a reference object. Let $x$ and $y$ be the directional relationcodes associated with object-pair $(A, C)$ and $(B, C)$, respectively. Then, the similarity between $x$ and $y$ is measured by the number of neighborhood areas spanned by $A \cap B$ divided by the number of neighborhood areas spanned by $A \cup B$. As a consequence, the directional similarity between two pictures $p$ and $q$ is first measured by summing up the directional similarity between $r_{1}$ and $r_{2}$ for all $r_{1}$ and $r_{2}$ (where $r_{1}$ is a directional relation in $p ; r_{2}$ is a directional relation in $q ; r_{1}$ and $r_{2}$ have the same target and reference objects, but are in two separate pictures) and then normalizing the measure by the total number of directional relations in both pictures. Thus, the directional similarity measure between $R_{p}$ and $R_{q}$ is defined as

$$
\begin{aligned}
& S_{D}\left(R_{p}, R_{q}\right)= \\
& \frac{\sum_{i=1}^{k} s_{D}\left(\varepsilon\left(t_{i}^{q}\right) \cdot D_{1}, t_{i}^{q} \cdot D_{1}\right)+\sum_{i=1}^{k} s_{D}\left(\varepsilon\left(t_{i}^{q}\right) \cdot D_{2}, t_{i}^{q} \cdot D_{2}\right)}{m+n},
\end{aligned}
$$

where

$$
s_{D}(x, y)= \begin{cases}1, & \text { if } \sum_{k=1}^{8} x(k) \vee y(k)=0 \\ \frac{\sum_{k=1}^{8} x(k) \wedge y(k)}{\sum_{k=1}^{8} x(k) \vee y(k)}, & \text { otherwise. }\end{cases}
$$

In the above formula, symbol " $\wedge$ " (“ $\vee$ ") represents the logical "AND" ("OR") operation. Similarly, we define the topological similarity measure between $R_{p}$ and $R_{q}$ as follows:

$$
S_{T}\left(R_{p}, R_{q}\right)=\frac{2 *\left(\sum_{i=1}^{k} s_{T}\left(\varepsilon\left(t_{i}^{q}\right) \cdot T, t_{i}^{q} \cdot T\right)\right)}{m+n},
$$

where

$$
s_{T}\left(r_{1}, r_{2}\right)=1-\frac{\left|r_{1}-r_{2}\right|}{4} .
$$

In (4), $r_{1}$ and $r_{2}$ are the codes for two topological relations. Thus, $\left|r_{1}-r_{2}\right|$ represents the distance between them. Our numbering scheme for topological relations reflects the degree of difference among all topological relations. For example, the distance between "disjoint" and "meet" is 1 , while the distance between "disjoint" and "partly_overlap" is 2. In a similar way of calculating directional similarity measure, (3) computes the topological similarity between two pictures $p$ and $q$ by considering all corresponding topological relations in both pictures and then normalizing the measure.

Notice that $S_{D}\left(R_{p}, R_{q}\right)$ and $S_{T}\left(R_{p}, R_{q}\right)$ are undefined if relation $\varepsilon$ does not exists (i.e., there is no object pair in query picture $q$ such that the same pair also appears in database picture $p$ ). Based on the above two similarity measuring

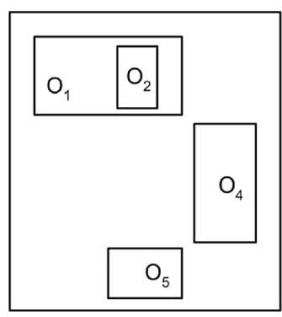

$p_{3}$

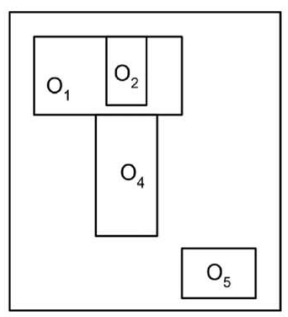

$p_{4}$ equations, we provide the following definitions for directional-similarity and topological-similarity.

Definition 1. A database picture $p$ is directionally similar to a query picture $q$ with a degree of similarity $s_{1}$ iff $S_{D}\left(R_{p}, R_{q}\right)=s_{1}$.

Definition 2. A database picture p is topological-similar to a query picture $q$ with a degree of similarity $s_{2}$ iff $S_{T}\left(R_{p}, R_{q}\right)=s_{2}$.

Now, we use the pictures shown in Fig. 7 to illustrate our similarity measures. The 9D-SPA representations for pictures $p_{1}, p_{2}, p_{3}$, and $p_{4}$ are listed below:

$$
\begin{aligned}
R_{p_{1}}=\{ & (1,255,0,4),(4,24,129,0),(5,24,129,0)\} . \\
R_{p_{2}}=\{ & (1,255,0,4),(4,8,128,0),(5,8,128,0)\} . \\
R_{p_{3}}=\{ & (1,255,0,4),(4,8,128,0),(7,12,64,0),(5,8,128,0), \\
& (8,4,224,0),(10,2,32,0)\} . \\
R_{p_{4}}=\{(1,241,0,3),(4,14,64,1),(7,8,128,0),(5,4,224,0), & (8,8,128,0),(10,8,128,0)\} .
\end{aligned}
$$

We arbitrarily pick up $R_{p_{3}}$ and $R_{p_{1}}$ to illustrate the process of calculating $S_{D}\left(R_{p_{3}}, R_{p_{1}}\right)$ and $S_{T}\left(R_{p_{3}}, R_{p_{1}}\right)$. After applying (2) to each tuple in $R_{p_{1}}$ and the corresponding tuple in $R_{p_{3}}$, we obtain the following results:

$$
\begin{array}{lll}
s_{D}\left(t_{1}^{p_{3}} \cdot D_{1}, t_{1}^{p_{1}} \cdot D_{1}\right)=1, & s_{D}\left(t_{2}^{p_{3}} \cdot D_{1}, t_{2}^{p_{1}} \cdot D_{1}\right)=1 / 2, & s_{D}\left(t_{4}^{p_{3}} \cdot D_{1}, t_{3}^{p_{1}} \cdot D_{1}\right)=1 / 2, \\
s_{D}\left(t_{1}^{p_{3}} \cdot D_{2}, t_{1}^{p_{1}} \cdot D_{2}\right)=1, & s_{D}\left(t_{2}^{p_{3}} \cdot D_{2}, t_{2}^{p_{1}} \cdot D_{2}\right)=1 / 2, & s_{D}\left(t_{4}^{p_{3}} \cdot D_{2}, t_{3}^{p_{1}} \cdot D_{2}\right)=1 / 2 .
\end{array}
$$

Thus, the similarity measure $S_{D}\left(R_{p_{3}}, R_{p_{1}}\right)$ is

$$
\begin{aligned}
& \frac{\sum_{i=1}^{3} s_{D}\left(\varepsilon\left(t_{i}^{p_{1}}\right) \cdot D_{1}, t_{i}^{p_{1}} \cdot D_{1}\right)+\sum_{i=1}^{3} s_{D}\left(\varepsilon\left(t_{i}^{p_{1}}\right) \cdot D_{2}, t_{i}^{p_{1}} \cdot D_{2}\right)}{3+6} \\
& =\frac{4}{9}=0.44 .
\end{aligned}
$$

By applying (4) to each tuple in $R_{p_{1}}$ and the corresponding tuple in $R_{p_{3}}$, we obtain the following results:

$$
s_{T}\left(t_{1}^{p_{3}} \cdot T, t_{1}^{p_{1}} \cdot T\right)=1, \quad s_{T}\left(t_{2}^{p_{3}} \cdot T, t_{2}^{p_{1}} \cdot T\right)=1, \quad s_{T}\left(t_{4}^{p_{3}} \cdot T, t_{3}^{p_{1}} \cdot T\right)=1 .
$$

Thus, the similarity measure $S_{T}\left(R_{p 3}, R_{p 1}\right)$ is

$$
\frac{2 *\left(\sum_{i=1}^{3} s_{T}\left(\varepsilon\left(t_{i}^{p_{1}}\right) \cdot T, t_{i}^{p_{1}} \cdot T\right)\right)}{3+6}=\frac{2 * 3}{9}=0.67 .
$$

Similarly, we calculate the similarity measures between picture $p_{1}$ and pictures $p_{2}$ and $p_{4}$ :

$$
\begin{array}{ll}
S_{D}\left(R_{p_{2}}, R_{p_{1}}\right)=0.67, & S_{T}\left(R_{p_{2}}, R_{p_{1}}\right)=1, \\
S_{D}\left(R_{p_{4}}, R_{p_{1}}\right)=0.24 & S_{T}\left(R_{p_{4}}, R_{p_{1}}\right)=0.56 .
\end{array}
$$




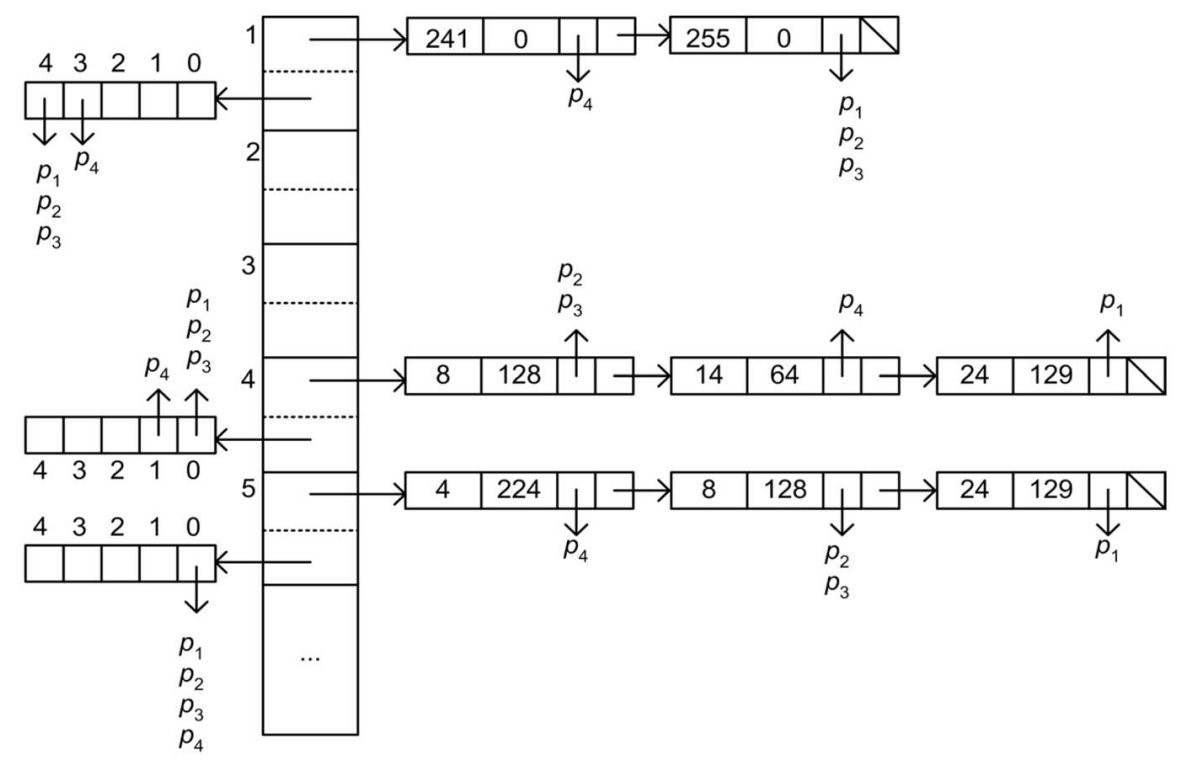

Fig. 8. An example of index structure for a pictorial database.

As a result, we can say that picture $p_{2}$ is directionally similar to picture $p_{1}$ with a degree of similarity 0.67 . Picture $p_{3}$ is directionally similar to picture $p_{1}$ with a degree of similarity 0.44 . Picture $p_{4}$ is directionally similar to picture $p_{1}$ with a degree of similarity 0.24 . Picture $p_{2}$ is topologicalsimilar to picture $p_{1}$ with a degree of similarity 1 . Picture $p_{3}$ is topological-similar to picture $p_{1}$ with a degree of similarity 0.67 . Picture $p_{4}$ is topological-similar to picture $p_{1}$ with a degree of similarity 0.56 . As compared to the pictures shown in Fig. 7, the similarity measures calculated by the system are consistent with what we expected.

\subsection{Index Structure}

The 9D-SPA representations of database pictures can be incorporated into a two-level index structure to facilitate image retrieval. An example of such an index structure is shown in Fig. 8. There are two levels of indices in this index structure based on 9D-SPA representation. The first-level index, called the level-1 index array, is an array of size $\frac{n(n-1)}{2}$, where $n$ is the number of distinct objects in the database. Each entry in this array may contain two pointers. One points to a list of directional relation-codes, called "direction-code list." The other points to an array of size 5, called "topological relation array." Each item in a direction-code list is an array of four elements: the first element is the directional relationcode $D_{i j}$; the second one is the directional relation-code $D_{j i}$; the third one is a pointer to a list of database images having directional relation-codes $D_{i j}$ and $D_{j i}$; the fourth one is a pointer to the next item in the current direction-code list. Each entry in a topological relation array corresponds to one type of topological relations and may contain a pointer to a list of database images. For example, in Fig. 8 , the tuple $(4,14,64,1)$ will be mapped to image $p_{4}$ according to our indexing scheme. In other words, image $p_{4}$ must contain objects $O_{1}$ and $\mathrm{O}_{4}$ because object-pair code 4 is decoded into object codes 1 and 4; Object $O_{1}$ is to the northeast or north or northwest of object $O_{4}$ because directional relation-code 14 is decoded into

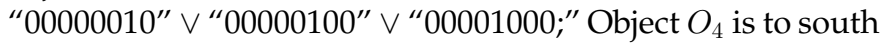
of object $O_{1}$ because directional relation-code 64 is decoded into "01000000;" and the topological relationship between objects $O_{1}$ and $O_{4}$ is "meet" because $T_{1,4}=1$.

\subsection{Image Retrieval Algorithm}

The index structure helps reducing the search space of query processing. The algorithm of retrieving similar images based on 9D-SPA representation and the two-level index structure is presented as follows:

Algorithm: Similarity retrieval.

Input: A 9D-SPA representation $R_{q}$ for query picture $q$ and two thresholds $h_{D}$ and $h_{T}$.

Output: $\left\{p \mid p\right.$ is a database picture such that $S_{D}\left(R_{p}, R_{q}\right) \geq h_{D}$ and $\left.S_{T}\left(R_{p}, R_{q}\right) \geq h_{T}\right\}$.

1. $\forall$ tuple $t=\left(O_{i j}, D_{i j}, D_{j i}, T_{i j}\right) \in R_{q}$

a. Find the direction-code list $L$ and topological relation array $M$ associated with $O_{i j}$ from the level-1 index array.

b. If $h_{D}=1$, then traverse the list $L$ to find the set $\Gamma_{t}^{\prime}$ of images associated with items $I$ in $L$ such that the two directional relation-codes in $I$ are $D_{i j}$ and $D_{i j}$.

c. If $h_{T}=1$, then obtain the set $\Gamma_{t}^{\prime \prime}$ of images associated with $M\left[T_{i j}\right]$.

d. If $h_{D} \neq 1$ and $h_{T} \neq 1$, then collect all the images pointed by $M[u]$ with $0 \leq u \leq 4$ and store them in $\Gamma_{t}^{\prime \prime}$.

2. Case of

a. $h_{D}=1$ and $h_{T}=1: \Gamma \leftarrow\left(\cap_{t \in R_{q}} \Gamma_{t}^{\prime}\right) \cap\left(\cap_{t \in R_{q}} \Gamma_{t}^{\prime \prime}\right)$.

b. $h_{D}=1$ and $h_{T} \neq 1: \Gamma \leftarrow \cap_{t \in R_{q}} \Gamma_{t}^{\prime}$.

c. $h_{D} \neq 1$ and $h_{T}=1: \Gamma \leftarrow \cap_{t \in R_{q}} \Gamma_{t}^{\prime \prime}$.

d. Otherwise: $\Gamma \leftarrow \cup_{t \in R_{q}} \Gamma_{t}^{\prime \prime}$.

3. If $h_{D} \neq 0$, then

$\forall p \in \Gamma$, if $\left(S_{D}\left(R_{p}, R_{q}\right)<h_{D}\right)$, then remove $p$ from $\Gamma$. 
4. If $h_{T} \neq 0$, then

$\forall p \in \Gamma$, if $\left(S_{T}\left(R_{p}, R_{q}\right)<h_{T}\right)$, then remove $p$ from $\Gamma$.

5. Sort $\Gamma$ by using $S_{D}\left(R_{p}, R_{q}\right)$ as the primary key and $S_{T}\left(R_{p}, R_{q}\right)$ as the secondary key in descending order.

6. Return $\Gamma$.

We use the index structure in Fig. 8 to illustrate our similarity retrieval algorithm. The index structure contains the pictures shown in Fig. 7. Assume that the 9D-SPA representation for query picture $q$ is $R_{q}=\left\{t_{1}, t_{2}, t_{3}\right\}=$ $\{(1,255,0,4),(4,24,129,0),(5,24,129,0)\}$ and the similarity requirement is $\left(h_{D}, h_{T}\right)=(1,1)$. Therefore, we obtain $\Gamma_{t_{1}}^{\prime}=$ $\left\{p_{1}, p_{2}, p_{3}\right\}, \Gamma_{t_{2}}^{\prime}=\left\{p_{1}\right\}, \Gamma_{t_{3}}^{\prime}=\left\{p_{1}\right\}, \Gamma_{t_{1}}^{\prime \prime}=\left\{p_{1}, p_{2}, p_{3}\right\}, \Gamma_{t_{2}}^{\prime \prime}=$ $\left\{p_{1}, p_{2}, p_{3}\right\}$, and $\Gamma_{t_{3}}^{\prime \prime}=\left\{p_{1}, p_{2}, p_{3}, p_{4}\right\}$, respectively. So, $\Gamma=$ $\left(\cap_{t \in R_{q}} \Gamma_{t}^{\prime}\right) \cap\left(\cap_{t \in R_{q}} \Gamma_{t}^{\prime \prime}\right)=\left\{p_{1}\right\}$. Since $S_{D}\left(R_{p_{1}}, R_{q}\right)=1$ and $S_{T}$ $\left(R_{p_{1}}, R_{q}\right)=1$, therefore, $p_{1}$ is the only qualified picture.

Now, we set the similarity requirement $\left(h_{D}, h_{T}\right)$ to $(0.6,0.5)$. Then, we obtain $\Gamma_{t_{1}}^{\prime \prime}=\Gamma_{t_{2}} \prime \prime=\Gamma_{t_{3}} \prime \prime=\left\{p_{1}, p_{2}, p_{3}, p_{4}\right\}$. Therefore, $\Gamma=\cup_{t \in R_{q}} \Gamma \prime_{t}=\left\{p_{1}, p_{2}, p_{3}, p_{4}\right\}$. The similarity measures $\left(S_{D}, S_{T}\right)$ for $p_{1}, p_{2}, p_{3}$, and $p_{4}$ with respect to $q$ are $(1,1)$, $(0.67,1),(0.44,0.67)$, and $(0.24,0.56)$, respectively. According to the current similarity requirement $\left(h_{D}, h_{T}\right)=(0.6,0.5)$, only pictures $p_{1}$ and $p_{2}$ are qualified and retrieved.

\section{EXPERIMENTAL RESULTS}

In this section, we present the simulation results to demonstrate the efficiency and effectiveness of our similarity retrieval system based on 9D-SPA representation and the two-level index structure. Without using an index structure, we need to inspect all 9D-SPA representations associated with the database images and compare them with the 9D-SPA representation of the query picture during image retrieval. By using the two-level indexing structure, the search space can be restricted to a small set of images. In the first part of our experiment, we would like to estimate the percentage of the average number of pictures to be accessed per query. We expect that this percentage will be very small if the index structure is used. In the second part of our experiment, we would like to demonstrate the performance of our image retrieval system in terms of recall and precision based on the knowledge structure of 9D-SPA representation.

\subsection{Efficiency of Image Retrieval}

In this part of the experiment, a database containing 50,000 pictures and 26 different iconic objects were created. There are three versions of databases. A picture in version-1 database contains four to six objects. A picture in version-2 database contains six to eight objects and a picture in version- 3 database contains eight to 10 objects. There are also three types of query pictures used for experiment. Type-1 picture contains two to three objects. Type- 2 picture contains three to four objects and type-3 picture contains four to five objects. The pictures were randomly generated by considering all possible directional and topological relations between objects.

We use the notation $\left[Q_{l}, Q_{u}\right]$ to represent the case that the number of objects contained in a query picture is between $Q_{l}$ and $Q_{u}$. Similarly, $\left[P_{l}, P_{u}\right]$ denotes that the number of objects contained in a database picture is between $P_{l}$ and $P_{u}$. There are nine test cases in this experiment:
TABLE 3

The Values of $p$ for Nine Test Cases against Four Similarity Requirements where $p=\frac{\text { Average Number of Pictures Accessed per Query }}{\text { Total Number of Pictures in the Database }} \times 100$ Percent

\begin{tabular}{|r|r|r|r|r|r|}
\hline \multirow{2}{*}[Q_{l},Q_{u}]{} & {$\left[P_{l}, P_{u}\right]$} & $(0,0)$ & $(1,0)$ & $(0,1)$ & $(1,1)$ \\
\cline { 3 - 6 } & {$[4,6]$} & 1.5569 & 0.1861 & 1.4210 & 0.1743 \\
& {$[6,8]$} & 3.1692 & 0.3971 & 2.9252 & 0.3745 \\
& {$[8,10]$} & 5.8012 & 0.5974 & 5.3409 & 0.5593 \\
\hline$[3,4]$ & {$[4,6]$} & 0.2494 & 0.0017 & 0.1860 & 0.0013 \\
& {$[6,8]$} & 0.9182 & 0.0078 & 0.8175 & 0.0070 \\
& {$[8,10]$} & 2.0102 & 0.0131 & 1.7330 & 0.0119 \\
\hline$[4,5]$ & {$[4,6]$} & 0.0849 & 0.0000 & 0.0678 & 0.0000 \\
& {$[6,8]$} & 0.3000 & 0.0000 & 0.2183 & 0.0000 \\
& {$[8,10]$} & 1.0564 & 0.0004 & 0.7138 & 0.0002 \\
\hline \multicolumn{2}{|r|}{ Average } & 1.6829 & 0.1337 & 1.4915 & 0.1254 \\
\hline
\end{tabular}

1. $\left[Q_{l}, Q_{u}\right]=[2,3]$ and $\left[P_{l}, P_{u}\right]=[4,6]$.

2. $\left[Q_{l}, Q_{u}\right]=[2,3]$ and $\left[P_{l}, P_{u}\right]=[6,8]$.

3. $\left[Q_{l}, Q_{u}\right]=[2,3]$ and $\left[P_{l}, P_{u}\right]=[8,10]$.

4. $\left[Q_{l}, Q_{u}\right]=[3,4]$ and $\left[P_{l}, P_{u}\right]=[4,6]$.

5. $\left[Q_{l}, Q_{u}\right]=[3,4]$ and $\left[P_{l}, P_{u}\right]=[6,8]$.

6. $\left[Q_{l}, Q_{u}\right]=[3,4]$ and $\left[P_{l}, P_{u}\right]=[8,10]$.

7. $\left[Q_{l}, Q_{u}\right]=[4,5]$ and $\left[P_{l}, P_{u}\right]=[4,6]$.

8. $\left[Q_{l}, Q_{u}\right]=[4,5]$ and $\left[P_{l}, P_{u}\right]=[6,8]$.

9. $\left[Q_{l}, Q_{u}\right]=[4,5]$ and $\left[P_{l}, P_{u}\right]=[8,10]$.

The similarity requirement of a query process is denoted by $\left(h_{D}, h_{T}\right)$, where $h_{D}$ is the threshold for directional-similarity and $h_{T}$ is the threshold for topological-similarity. The similarity requirement $\left(h_{D}, h_{T}\right)=(0,0)$ means that a database picture $p$ is similar to the query picture $q$ if some objectpair in $q$ can also be found in $p$ without considering the spatial relationships among objects in both pictures. The similarity requirement $\left(h_{D}, h_{T}\right)=(0,1)$ means that $q$ and $p$ have the same objects and all topological relationships between objects in the query picture must be fully matched with those in a relevant database picture. The similarity requirement $\left(h_{D}, h_{T}\right)=(1,0)$ means that $q$ and $p$ have the same objects and all directional relationships between objects in the query picture are matched with those in a relevant database picture. For $\left(h_{D}, h_{T}\right)=(1,1)$, two pictures are considered as similar only if they have the same objects and all the spatial relationships (both directional and topological) between objects in the query picture are fully matched with those in the database picture.

In our experiment, we tested nine cases against four similarity requirements as shown in Table 3 . For each case and each similarity requirement, we randomly generated 100 query pictures and calculated the average number of database pictures accessed per query. Each data entry shown in Table 3 is the average number of database pictures accessed per query divided by the total number of database pictures. It can be seen that the percentages are very low for all test cases and similarity requirements. For example, in 
TABLE 4

The System's Performance in Terms of Recall and Precision by Considering Directional-Similarity $\left(h_{T}=0\right)$

\begin{tabular}{|c|r|r|c|c|c|c|c|c|c|}
\hline \multirow{2}{*}[Q_{l},Q_{u}]{} & \multicolumn{2}{|c|}{$h_{D}=0.2$} & \multicolumn{2}{c|}{$h_{D}=0.4$} & \multicolumn{2}{c|}{$h_{D}=0.6$} & \multicolumn{2}{c|}{$h_{D}=0.8$} \\
\cline { 3 - 10 } & {$\left[P_{l}, P_{u}\right]$} & Rec. & Pre. & Rec. & Pre. & Rec. & Pre. & Rec. & Pre. \\
\hline$[2-3]$ & {$[2-4]$} & 100.0 & 43.0 & 77.4 & 54.5 & 65.1 & 94.7 & 64.0 & 99.2 \\
\hline$[3-4]$ & {$[3-7]$} & 100.0 & 10.5 & 83.1 & 77.8 & 80.2 & 96.0 & 80.0 & 100.0 \\
\hline$[3-4]$ & {$[3-10]$} & 81.2 & 15.9 & 77.1 & 87.5 & 70.7 & 96.5 & 68.5 & 100.0 \\
\hline \multicolumn{2}{|c|}{ average } & 93.7 & 23.1 & 79.2 & 73.3 & 72.0 & 95.7 & 70.8 & 99.7 \\
\hline
\end{tabular}

the case of $\left[Q_{l}, Q_{u}\right]=[2,3]$ and $\left[P_{l}, P_{u}\right]=[8,10]$, the percentage of the average number of database pictures accessed per query with similarity requirement $\left(h_{D}, h_{T}\right)=(0,0)$ is 5.8012 percent. Moreover, similarity requirement $\left(h_{D}, h_{T}\right)=$ $(0,0)$ represents the fuzziest measure while similarity requirement $\left(h_{D}, h_{T}\right)=(1,1)$ represents the most precise measure. The average percentages of images accessed per query for all nine cases from $\left(h_{D}, h_{T}\right)=(0,0)$ to $\left(h_{D}, h_{T}\right)=$ $(1,1)$ are 1.6829 percent, 0.1337 percent, 1.4915 percent, and 0.1254 percent, respectively. As we can see, only a very small portion of database images are accessed per query based on our two level index structure, thus the efficiency of similarity retrieval is well demonstrated.

\subsection{Effectiveness of Image Retrieval}

In this part of the experiment, we measured the effectiveness of our image retrieval system in terms of recall and precision. For a given query picture, let $a$ be the number of all relevant pictures, $b$ be the number of pictures that are relevant and retrieved, and $c$ be the number of pictures retrieved. Then, recall $=\frac{b}{a} \times 100$ percent and precision $=$ $\frac{b}{c} \times 100$ percent. First, we consider directional-similarity retrieval (i.e., $h_{T}=0$ ). The three test cases are:

1. $\left[Q_{l}, Q_{u}\right]=[2,3]$ and $\left[P_{l}, P_{u}\right]=[2,4]$.

2. $\left[Q_{l}, Q_{u}\right]=[3,4]$ and $\left[P_{l}, P_{u}\right]=[3,7]$.

3. $\left[Q_{l}, Q_{u}\right]=[3,4]$ and $\left[P_{l}, P_{u}\right]=[3,10]$.

As we can see from Table 4 , recall is decreasing while precision is increasing as $h_{D}$ increases from 0.2 to 0.4 , to 0.6 , then to 0.8 for all three test cases. When $h_{D}=0.2$, the recall reaches 93.7 percent with 23.1 percent precision rate on an average. This is because the similarity requirement was set to a very low value. However, as $h_{D}$ increases to 0.8 , the precision rate reaches 99.7 percent, while the recall rate still maintains at 70.8 percent on average.

Next, we evaluate the recall and precision by considering topological-similarity retrieval (i.e., $h_{D}=0$ ). There are also three test cases:

1. $\left[Q_{l}, Q_{u}\right]=[2,3]$ and $\left[P_{l}, P_{u}\right]=[2,4]$.

2. $\left[Q_{l}, Q_{u}\right]=[3,4]$ and $\left[P_{l}, P_{u}\right]=[2,4]$.

3. $\left[Q_{l}, Q_{u}\right]=[3,4]$ and $\left[P_{l}, P_{u}\right]=[3,7]$.

Table 5 shows that recall is decreasing while precision is increasing as $h_{T}$ is increased from 0.5 to 0.6 , to 0.8 , then to 0.9 for all three test cases. As $h_{T}$ increases to 0.9 , the precision rate increases to 94.4 percent and the recall rate drops from 82.6 percent to 51.0 percent on an average.

\section{Conclusions}

Content-based image retrieval (CBIR) based on visual features of images is the trend of designing a modern image database system. In the past, symbolic pictures are used to approximate segmented images containing recognized objects. Searching for desired images based on symbolic pictures is called "iconic indexing." The most important feature of a symbolic picture is probably the binary spatial relationships between objects. Thus, an

TABLE 5

The System's Performance in Terms of Recall and Precision by Considering Topological-Similarity $\left(h_{D}=0\right)$

\begin{tabular}{|c|r|r|l|l|l|l|l|l|l|}
\hline \multirow{2}{*}[Q_{l},Q_{u}]{} & \multicolumn{2}{|c|}{$h_{T}=0.5$} & \multicolumn{2}{c|}{$h_{T}=0.6$} & \multicolumn{2}{c|}{$h_{T}=0.8$} & \multicolumn{2}{c|}{$h_{T}=0.9$} \\
\cline { 3 - 10 } & {$\left[P_{l}, P_{u}\right]$} & Rec. & Pre. & Rec. & Pre. & Rec. & Pre. & Rec. & Pre. \\
\hline$[2-3]$ & {$[2-4]$} & 73.6 & 50.7 & 55.8 & 77.7 & 38.1 & 85.9 & 38.1 & 95.8 \\
\hline$[3-4]$ & {$[2-4]$} & 100.0 & 12.5 & 100.0 & 45.2 & 77.8 & 75.0 & 77.8 & 90.5 \\
\hline$[3-4]$ & {$[3-7]$} & 74.3 & 39.9 & 61.7 & 59.5 & 49.1 & 92.3 & 37.2 & 97.0 \\
\hline \multicolumn{2}{|c|}{ average } & 82.6 & 34.4 & 72.5 & 60.8 & 55.0 & 84.4 & 51.0 & 94.4 \\
\hline
\end{tabular}


appropriate knowledge representation for spatial relations plays an important role in designing a CBIR system.

A novel image representation called 9D-SPA has been presented in this paper to capture the information about the spatial relationships between objects in a segmented picture. The capability of representing a picture using 9D-SPA representation is more powerful than any other representation schemes based on MBR or centroids of objects. The 9D-SPA representation well supports visualization and browsing, spatial reasoning, as well as similarity retrieval. The flexibility of similarity measures are provided to support fuzzy matching that is highly desired to meet user's different requirements. By exercising a database containing 50,000 pictures, we successfully demonstrated the efficiency and effectiveness of our image retrieval system based on 9D-SPA representation and a two-level index structure.

\section{ACKNOWLEDGMENTS}

This research work was supported by the project NSC902213-E-005-015 granted by the National Science Council of the Republic of China. The authors would also like to thank all the anonymous reviewers of this paper for their invaluable suggestions and comments.

\section{REFERENCES}

[1] J.R. Bach, C. Fuller, A. Gupta, A. Hampapur, B. Horowitz, R. Humphrey, R.C. Jain, and C. Shu, "Virage Image Search Engine: An Open Framework for Image Management," Proc. Symp. Electronic Imaging: Science and Technology-Storage \& Retrieval for Image and Video Database IV, pp. 76-87, 1996.

[2] B. Bhanu and S. Lee, Genetic Learning for Adaptive Image Segmentation. Norwell: Kluwer Academic, 1994.

[3] C.C. Chang, "Spatial Match Retrieval of Symbolic Pictures," J. Information Science and Eng., vol. 7, pp. 405-422, Dec. 1991.

[4] S.K. Chang, E. Jungert, and Y. Li, "Representation and Retrieval of Symbolic Pictures Using Generalized 2D Strings," technical report, Univ. of Pittsburg, 1988.

[5] S.K. Chang, Q.Y. Shi, and C.W. Yan, "Iconic Indexing by 2-D Strings," IEEE Trans. Pattern Analysis and Machine Intelligence, vol. 9, no. 3, pp. 413-428, May 1987.

[6] S.K. Chang, Principles of Pictorial Information Systems Design. Englewood Cliffs, N.J.: Prentice-Hall Inc., 1989.

[7] Y. Chen and J.Z. Wang, "A Region-Based Fuzzy Feature Matching Approach to Content-Based Image Retrieval," IEEE Trans. Pattern Analysis and Machine Intelligence, vol. 24, no. 9, pp. 1252-1267, Sept. 2002.

[8] M. Flickner, H. Sawhney, W. Niblack, J. Ashley, Q. Huang, B. Dom, M. Gorkani, J. Hafner, D. Lee, D. Petkovic, D. Streele, and P. Yanker, "Query by Image and Video Content: The QBIC System," Computer, vol. 28, no. 9, pp. 23-32, Sept. 1995.

[9] P.W. Huang and Y.R. Jean, "Using 2D $\mathrm{PC}^{+}$-Strings as Spatial Knowledge Representation for Image Database Systems," Pattern Recognition, vol. 27, no. 9, pp. 1249-1257, Sept. 1994.

[10] P.W. Huang and Y.R. Jean, "Design of Large Intelligent Image Database Systems," Int'l J. Intelligent Systems, vol. 11, pp. 347-365, 1996.

[11] P.W. Huang and S.K. Dai, "Image Retrieval by Texture Similarity," Pattern Recognition, vol. 36, pp. 665-679, 2003.

[12] E. Jungert, "Extended Symbolic Projections as a Knowledge Structure for Spatial Reasoning," Proc. Fourth BPRA Conf. Pattern Recognition, pp. 343-351, 1988.

[13] E. Jungert and S.K. Chang, "An Algebra for Symbolic Image Manipulation and Transformation," Visual Database Systems, T.L. Kunii, ed., North Holland: Elsevier Science Publishers B.V., 1989.

[14] L.J. Latecki and R. Lakamper, "Application of Planar Shape Comparison to Object Retrieval in Image Database," Pattern Recognition, vol. 35, pp. 15-29, 2002.

[15] S.Y. Lee and F.J. Hsu, "2D C-String: A New Spatial Knowledge Representation for Image Database Systems," Pattern Recognition, vol. 23, no. 10, pp. 1077-1087, Oct. 1990.
[16] S.Y. Lee and F.J. Hsu, "Spatial Reasoning and Similarity Retrieval of Images Using 2D C-String Knowledge Representation," Pattern Recognition, vol. 25, no. 3, pp. 305-318, Mar. 1992.

[17] K.C. Liang and C.C. Jay Kuo, "WaveGuide: A Joint Wavelet-Based Image Representation and Description System," IEEE Trans. Image Processing, vol. 8, no. 11, pp. 1619-1629, 1999.

[18] A.K. Majumdar, I. Bhattacharya, and A.K. Saha, "An ObjectOriented Fuzzy Data Model for Similarity Detection in Image Databases," IEEE Trans. Knowledge and Data Eng., vol. 14, no. 5, pp. 1186-1189, Sept./Oct. 2002.

[19] M. Nabil, J. Shepherd, and A.H.H. Ngu, "2D Projection Interval Relationships: A Symbolic Representation of Spatial Relationships," Lecture Notes in Computer Science, no. 951, pp. 292-309, 1995.

[20] M. Nabil, A.H.H. Ngu, and J. Shepherd, "Picture Similarity Retrieval Using the 2D Projection Interval Representation," IEEE Trans. Knowledge and Data Eng., vol. 8, no. 4, pp. 533-539, Aug. 1996.

[21] A. Pentland, R.W. Picard, and S. Sclaroff, "Photobook: Tool for Content-Based Manipulation of Image Databases," Int'l J. Computer Vision, vol. 18, no. 3, pp. 233-254, June 1996.

[22] G. Petraglia, M. Sebillo, M. Tucci, and G. Tortora, "Virtual Images for Similarity Retrieval in Image Databases," IEEE Trans. Knowledge and Data Eng., vol. 13, no. 6, pp. 951-967, Nov./Dec. 2001.

[23] E. Petrakis, C. Faloutsos, and K.I. Lin, "ImageMap: An Image Indexing Method Based on Spatial Similarity," IEEE Trans. Knowledge and Data Eng., vol. 14, no. 5, pp. 979-987, Sept./Oct. 2002.

[24] A. Rao, R.K. Srihari, L. Zhu, and A. Zhang, "A Method for Measuring the Complexity of Image Databases," IEEE Trans. Multimedia, vol. 4, no. 2, pp. 160-173, Mar./Apr. 2002.

[25] Y. Rui, T.S. Huang, "Image Retrieval: Current Techniques, Promising Directions, and Open Issues," J. Visual Comm. Image Representation, vol. 10, pp. 39-62, 1999.

[26] J.R. Smith and S.F. Chang, "VisualSEEK: A Full Automated Content-Based Image Query System," Proc. Fourth ACM Int'l Multimedia Conf., pp. 87-98, 1996.

[27] J. Vleugels, R.C. Veltkamp, and C. Remco, "Efficient Image Retrieval through Vantage Objects," Pattern Recognition, vol. 35, pp. 69-80, 2002.

[28] X.M. Zhou and C.H. Ang, "Retrieving Similar Pictures from a Pictorial Database by an Improved Hashing Table," Pattern Recognition Letters, vol. 18, pp. 751-758, 1997.

[29] http://www.annapolistech.com/reseller/retrieval.htm, 2004.

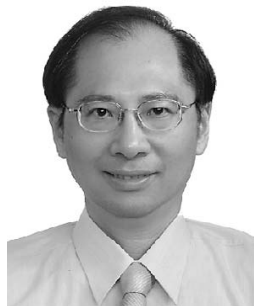

Po-Whei Huang received the BS degree in applied mathematics from National Chung-Hsing University in 1973, the MS degree in mathematics from Texas Tech University in 1978, and the $\mathrm{PhD}$ degree in computer science from Southern Methodist University in 1989. He was with Texas Instruments in Dallas as a member of technical staff and software development manager from 1978 to 1990 . He was the department head and has been a professor in the Computer Science Department at National Chung-Hsing University. Since September of 2002, he has been serving as the vice president of National Huwei University of Science and Technology located in Yunlin County of Taiwan. His research interests are in the fields of image database, visual inspection, pattern recognition, and artificial intelligence.

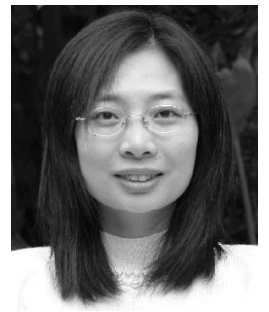

Chu-Hui Lee received the BS degree and MS degree in applied mathematics, both from National Chengchi University, in 1990 and 1992, respectively. She has been a lecturer in the Department of Information Management at Chaoyang University of Technology since 1994. Her current research interests are in the areas of image database and information retrieval.

$\triangleright$ For more information on this or any other computing topic, please visit our Digital Library at www.computer.org/publications/dlib. 